\title{
Reliability of Finger Photoplethysmogram Assessment: Effect of Mental Stress and Day-to-Day Variability
}

\author{
Marina Fukuie ${ }^{1,2}$, Takashi Tarumi², Takayuki Yamabe ${ }^{1,2}$, Jun Sugawara ${ }^{1,2 *}$, (D) \\ ${ }^{1}$ Graduate School of Comprehensive Human Sciences, University of Tsukuba, 1-1-1 Tennodai, Tsukuba, Ibaraki 305-8564, Japan \\ ${ }^{2}$ Human Informatics and Interaction Research Institute, National Institute of Advanced Industrial Science and Technology (AIST), \\ 1-1-1 Higashi, Tsukuba, Ibaraki 305-8566, Japan
}

\section{ARTICLE INFO}

Article History

Received 02 October 2020

Accepted 11 January 2021

Keywords

Arterial stiffness

augmentation index

finger photoplethysmogram

aging

cardiovascular disease risk

\begin{abstract}
Frequent assessment of vascular health (e.g., arterial stiffness) may predict the incidence of cardiovascular disease. The Second Derivative of the finger Photoplethysmogram Aging Index (SDPTGAI) may be used as an index of vascular aging and a screening tool for arteriosclerotic disease. To test its reliability as a measurement of arterial stiffness, we compared SDPTGAI with aortic Augmentation Index (AIx) at rest and during short-term mental stress (study 1). In addition, the day-to-day variability of SDPTGAI was evaluated (study 2). In study 1 , a total of 31 apparently healthy adults were measured with the photoplethysmogram on the right index finger in the sitting resting condition. Simultaneously, the applanation tonometry was used to record blood pressure waveforms from the left radial artery (for aortic pressure waveform estimation via a general transfer function). Subsequently, 22 subjects underwent a 30-s arithmetic test (double-digit subtraction). In study 2, 10 young adults were measured with SDPTGAI for five consecutive days. In study 1, we found that SDPTGAI was significantly correlated with AIx $(r=0.682, p<0.001)$. During the arithmetic task, heart rate and SDPTGAI were significantly increased from the baseline values ( $p<0.05$ for both) whereas AIx remained to be unchanged. In study 2, SDPTGAI showed $12.0 \pm 9.9 \%$ of Coefficient of Variation (CV) across all subjects, which is comparable to CV of heart rate (12.5 $\pm 5.4 \%)$. These findings suggest that SDPTGAI has the potential for evaluating arterial stiffness and wave reflection at the resting condition, but it needs to be measured under well-rested relaxed condition because the finger photoplethysmogram may easily be affected by mental stressor.
\end{abstract}

(C) 2021 The Authors. Publishing services by Atlantis Press International B.V. This is an open access article distributed under the CC BY-NC 4.0 license (http://creativecommons.org/licenses/by-nc/4.0/).

\section{INTRODUCTION}

The frequent assessment of vascular health may contribute to increased awareness and prevention of cardiovascular events in general populations. It has been well recognized that arterial stiffness and wave reflection measured by the aortic pulse wave velocity and Augmentation Index (AIx) are the strong predictors of future Cardiovascular events and all-cause mortality $[1,2]$. However, these measures require technical expertise and knowledge. In this context, the Second Derivative waveform of fingertip Photoplethysmography (SDPTG) can be measured easily from a fingertip placed on the photoplethysmography (PTG) sensor and has the potential for selfmonitoring of vascular health during daily living [3]. Previously, several studies have demonstrated clinical and pathophysiological importance of SDPTG indices such as the SDPTG Aging Index (SDPTGAI) [4-6]. Since SDPTGAI is strongly correlated with age, it can be rescaled to years of age which is easily comprehended by general populations [3]. For these reasons, SDPTG indices including the vascular aging index have recently been implemented for ambulatory monitoring devices, but its usability and reliability are not well documented.

Corresponding author.Email: jun.sugawara@aist.go.jp

Peer review under responsibility of the Association for Research into Arterial Structure and Physiology

Data availability statement: The data that support the findings of this study are available

from the corresponding author [JS], upon reasonable request.
Mental stress is an external stimulus that can affect physiological parameters such as arterial tone [5,7], blood viscosity [8], and heart rate [9]. It is reported that pulse wave velocity and AIx are increased with acute mental stress $[10,11]$. Moreover, skin blood flow shows transient reflex changes in response to various external stimuli (e.g., arousing or painful stimuli, emotional stress) [12]. Since photoplethysmogram optically evaluates blood volume changes in the microvascular bed of cutaneous tissue, SDPTG may be influenced by mental stressor independent of changes in vascular tone and stiffness of the relatively proximal vessels.

Therefore, the purpose of this study was to determine the usability and reliability of SDPTG indices. Specifically, we hypothesized that SDPTGAI is positively correlated with aortic AIx at rest and during short-term mental stress. Also, we investigated the day-today variability of SDPTG indices.

\section{MATERIALS AND METHODS}

\subsection{Subjects and Protocol}

In study 1 , a total of 31 healthy adults (11 men and 20 women, $41 \pm 2$ years of mean age) were studied. In study 2, 10 young healthy adults (eight men and two women, $24 \pm 2$ years of mean age) 
were measured SDPTG for 4-5 consecutive days in the morning (at the same time of day as much as possible). All experimental procedures and protocols conformed to the Declaration of Helsinki and were approved by the Institutional Review Board (\#2016-695, National Institute of Advanced Industrial Science and Technology). All subjects provided written informed consent before participation.

\subsection{Measurements and Analysis}

Study 1. After 5 min of quiet resting, all subjects underwent simultaneous recordings of the photoplethysmogram on the right index finger and the applanation tonometry to record blood pressure waveforms from the left radial artery in the sitting posture (resting). Then, a subgroup of subjects $(n=22)$ underwent mental arithmetic test which consisted of a control trial followed by an arithmetic trial (non-random order) with approximately a 5-min interval. In the control trial, participants read out two-digit numbers presented every $2 \mathrm{~s}$ on a computer screen for $30 \mathrm{~s}$. In the arithmetic trial, participants verbally answered two-digit subtractions presented every $2 \mathrm{~s}$ on a computer screen for 30 seconds.
The intermittent brachial cuff and continuous radial blood pressure waveforms were recorded from the left arm during each trial using a vascular screening device (HEM-9010AI, Omron Healthcare, Kyoto, Japan). The brachial pressure was measured by the oscillometric method while the radial pressure waveforms were recorded by applanation tonometry (HEM-9010AI, Omron Healthcare). The SDPTG was collected from the right index finger using a customized finger PTG device (Alps, Tokyo, Japan). All of the measurements above were simultaneously recorded during the resting and task periods. Radial artery pressure waveforms were converted to aortic pressure waveforms via a validated generalized transfer function (SphygmoCor software, AtCor Medical, Sydney, Australia) as previously reported [13]. AIx was calculated as pressure wave above its systolic shoulder divided by pulse pressure [14]. AIx was adjusted for $75 \mathrm{bpm}$ of heart rate $\left(\mathrm{AIx}_{\mathrm{HR75}}\right)$ was also obtained because it is affected by changes in heart rate independent of arterial stiffness and wave refection [15]. Heart rate was estimated from the averaged pulse interval.

Figure 1 presents sample PTG signals of the resting and the mental stress conditions. As shown in bottom panels, the SDPTG is characterized by five inflection points on finger PTG waves: the initial

(1)

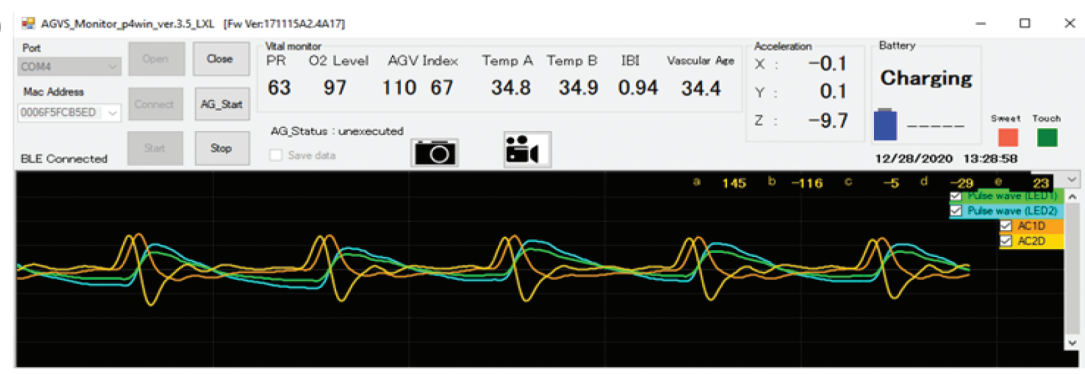

(2)

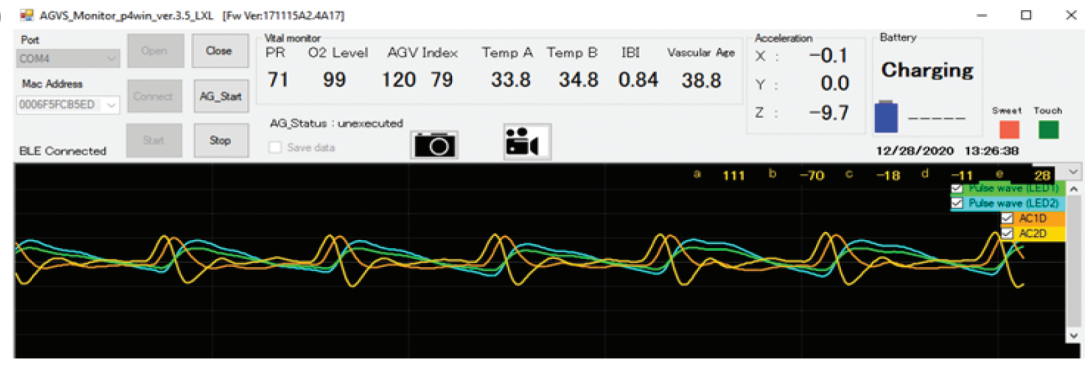

(3)

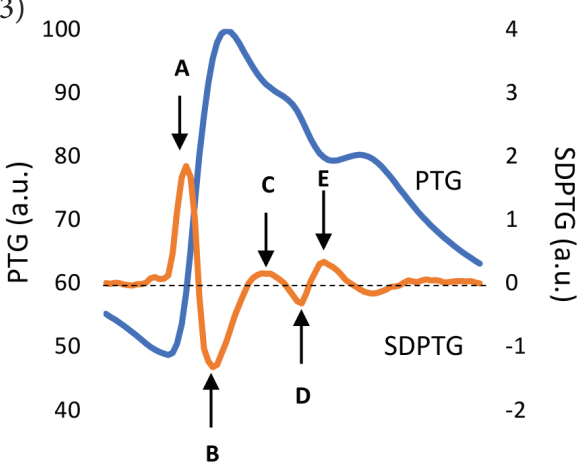

(4) 100

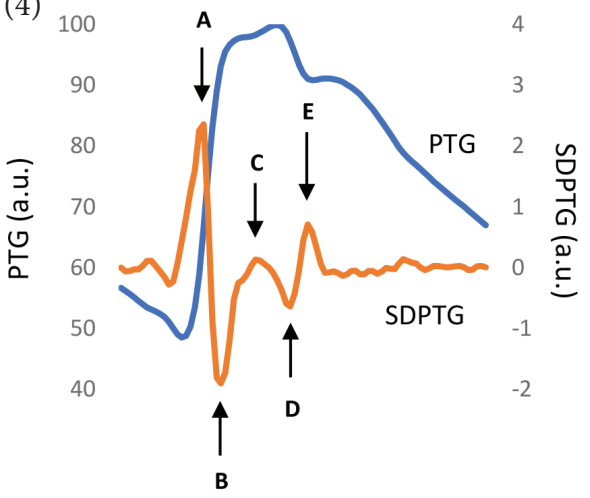

Figure 1 Samples of finger Photoplethysmogram (PTG) during the resting (1) and the mental stress (2) conditions. Bottom panels indicate synthesized 1-beat PTG (blue lines) and the Second Derivative of the finger Photoplethysmogram (SDPTG, orange lines) during the resting (3) and the mental stress (4) conditions, respectively. A-E are five inflection points on finger PTG waves characterized by the SDPTG: the initial positive (A), early negative (B), re-increasing (C), late re-decreasing (D), and diastolic positive waves (E). a.u. indicates arbitrary unit. 
positive (A), early negative (B), re-increasing (C), late re-decreasing (D), and diastolic positive waves (E), respectively. Absolute values of these waves' heights were obtained and used to calculate $\mathrm{B} / \mathrm{A}$ ratio, $\mathrm{D} / \mathrm{A}$ ratio, and SDPTGAI $(=[\mathrm{B}-\mathrm{C}-\mathrm{D}-\mathrm{E}] / \mathrm{A})$ as previously reported [3]. The higher $\mathrm{B} / \mathrm{A}$ ratio (= lower negative ratio) suggests low elasticity, vasoconstriction, and arteriosclerosis [5]. The D/A ratio is closely related to the augmentation of blood pressure in the aorta by wave reflection, an index to assess left ventricular (LV) afterload [5].

Study 2. To investigate the day-to-day variability of SDPG indices, each subject underwent measurement of SDPTG after a 5-min quiet resting for 4-5 consecutive days in the morning (at the same time of day as much as possible).

\subsection{Statistical Analysis}

In study 1 , intra-observer reproducibility of the SDPTG indices and aortic AIx was examined by Intraclass Correlation (ICC) between baseline measurements of the control and arithmetic conditions. A simple correlation analysis was performed to determine the relationship between variables of interest. Baseline variables were compared by paired $t$-test. A generalized linear model of repeated measures analysis of variance was used to determine the interaction effect of condition (i.e., control vs. mental arithmetic) and time (i.e., pre vs. during task). In case of a significant $F$-test, post-hoc pairwise Bonferroni comparisons were performed. In study 2 , the day-to-day variability of $\mathrm{B} / \mathrm{A}$ ratio, $\mathrm{D} / \mathrm{A}$ ratio, and SDPTGAI were evaluated by calculating the Coefficient of Variation (CV) from the Standard Deviation (SD) and average values. As a secondary analysis, each CV was compared between two groups based on the time of measurement (before 9:00 am vs. after 11:00 am). Data are presented as mean and SD. Statistical significance was set $a$ priori at $p<0.05$. All data analyses were performed using a commercial software (SPSS, Version 25, IBM Corp., Armonk, NY, USA).

\section{RESULTS}

Study 1. Excellent intra-observer reproducibility was observed on the $\mathrm{B} / \mathrm{A}$ ratio $(\mathrm{ICC}=0.868), \mathrm{D} / \mathrm{A}$ ratio $(\mathrm{ICC}=0.793)$, SDPTGAI $(\mathrm{ICC}=0.914)$, and AIx $(\mathrm{ICC}=0.844)(p<0.001$ for all $)$. Figure 2 depicts relationships between AIx and SDPTG indices. The B/A ratio $(r=-0.531), \mathrm{D} / \mathrm{A}$ ratio $(r=0.740)$, and SDPTGAI $(r=0.682)$ were significantly correlated with AIx $(p<0.001$ for all).

There were no significant differences in the baseline blood pressure, heart rate, SDPTG indices, $\mathrm{AIx}$, and $\mathrm{AIx}_{\mathrm{HR} 75}$ between the control and arithmetic trials (Table 1). As shown in Figure 3, heart rate significantly increased during the control $(+3.6 \pm 5.2 \mathrm{bpm}, p=0.008)$ and arithmetic $(+8.4 \pm 4.9 \mathrm{bpm}, p<0.001)$ tasks from their corresponding baseline values. Moreover, heart rate during the arithmetic task was significantly higher than that during the control task $(p=0.001)$. During the arithmetic task, D/A ratio significantly increased from the baseline $(p<0.001)$. SDPTGAI also significantly increased from the baseline $(p=0.002)$ and was significantly higher than that during the control task $(p=0.006)$. The $\mathrm{B} / \mathrm{A}$ ratio significantly decreased by $9 \%$ from the baseline $(p<0.001)$ during the arithmetic task and was significantly lower than that during the control task $(p=0.004)$. Significant interaction between trial and time was not seen in either $\operatorname{AIx}(p=0.437)$ or $\operatorname{AIx}_{\mathrm{HR} 75}(p=0.351)$.

Study 2. Mean and SD of heart rate and SDPTG indices for five consecutive days were summarized in Table 2. CV of pulse rate, $\mathrm{B} / \mathrm{A}$ ratio, D/A ratio, and SDPTGAI were calculated in each subject. The average CV across all subjects were $12.5 \pm 5.4 \%$ for pulse rate, $10.6 \pm 5.9 \%$ for $\mathrm{B} / \mathrm{A}$ ratio, $128.7 \pm 139.9 \%$ for $\mathrm{D} / \mathrm{A}$ ratio, and $12.0 \pm 9.9 \%$ for SDPTGAI. When subjects were divided into two

Table 1 Baseline hemodynamic variables, augmentation index, and the Second Derivative finger Photoplethysmogram (SDPTG) indices

\begin{tabular}{|c|c|c|c|}
\hline Variables & Control trial & Arithmetic trial & $p$-values \\
\hline Heart rate, bpm & $76 \pm 7$ & $75 \pm 8$ & 0.936 \\
\hline Systolic BP, mmHg & $121 \pm 10$ & $121 \pm 10$ & 0.976 \\
\hline Mean BP, mmHg & $91 \pm 10$ & $91 \pm 9$ & 0.923 \\
\hline Diastolic BP, mmHg & $74 \pm 10$ & $74 \pm 9$ & 0.923 \\
\hline $\mathrm{AIx}, \%$ & $22.0 \pm 10.1$ & $21.8 \pm 12.1$ & 0.969 \\
\hline $\mathrm{AIx}_{\mathrm{HR} 75}, \%$ & $21.9 \pm 9.7$ & $21.8 \pm 10.8$ & 0.972 \\
\hline $\mathrm{B} / \mathrm{A}$ ratio, ratio & $0.79 \pm 0.13$ & $0.82 \pm 0.14$ & 0.493 \\
\hline $\mathrm{D} / \mathrm{A}$ ratio, ratio & $0.33 \pm 0.16$ & $0.29 \pm 0.14$ & 0.404 \\
\hline SDPTGAI, a.u. & $-0.72 \pm 0.36$ & $-0.77 \pm 0.36$ & 0.635 \\
\hline
\end{tabular}

Data are mean and SD. $p$-values were obtained by the paired $t$-test. BP, blood pressure; AIx, augmentation index; $\mathrm{AIx}_{\mathrm{HR75}}$, AIx adjusted by $75 \mathrm{beat} / \mathrm{min}$ of heart rate; $\mathrm{B} / \mathrm{A}$ ratio, the ratio of the absolute height of the early negative wave to that of the initial positive wave of SDTPG; D/A ratio, the ratio of the absolute height of the late re-decreasing wave to that of the initial positive wave of SDTPG; SDPTGAI, SDPTG aging index.
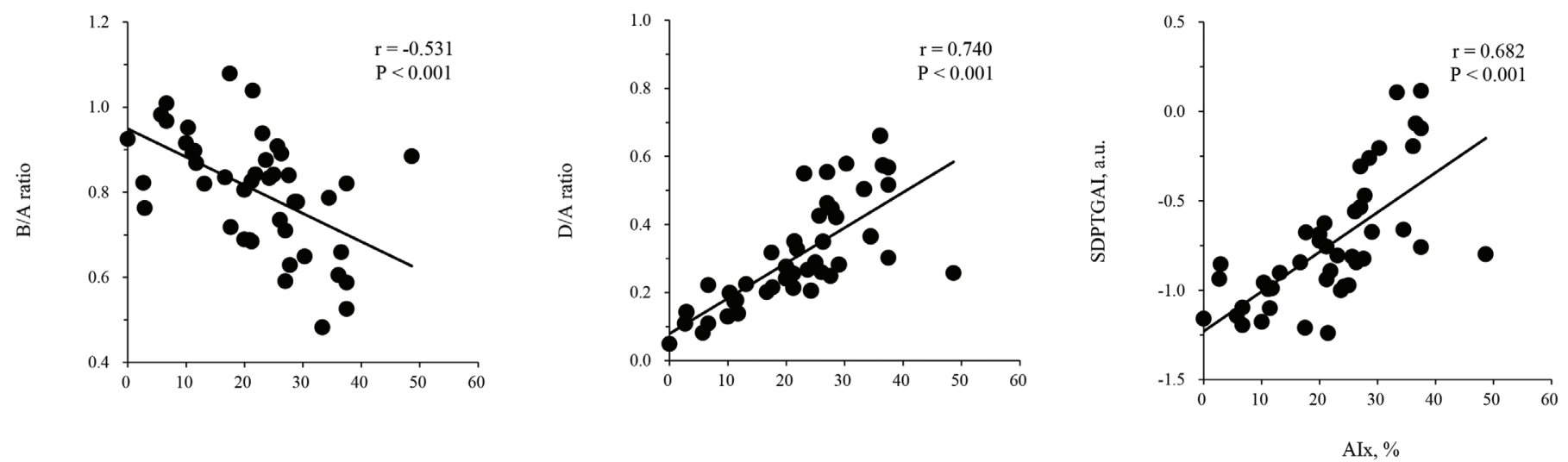

Figure 2 Scatterplots of Augmentation Index (AIx) and the Second Derivative of the finger Photoplethysmogram (SDPTG) indices. a.u. indicates arbitrary unit. 

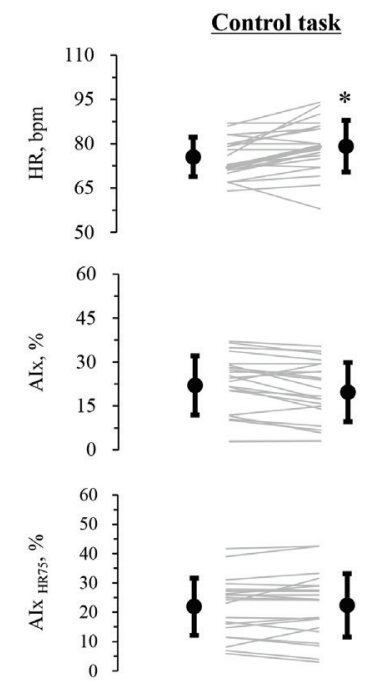

$\underline{\text { Arithmetic task }}$
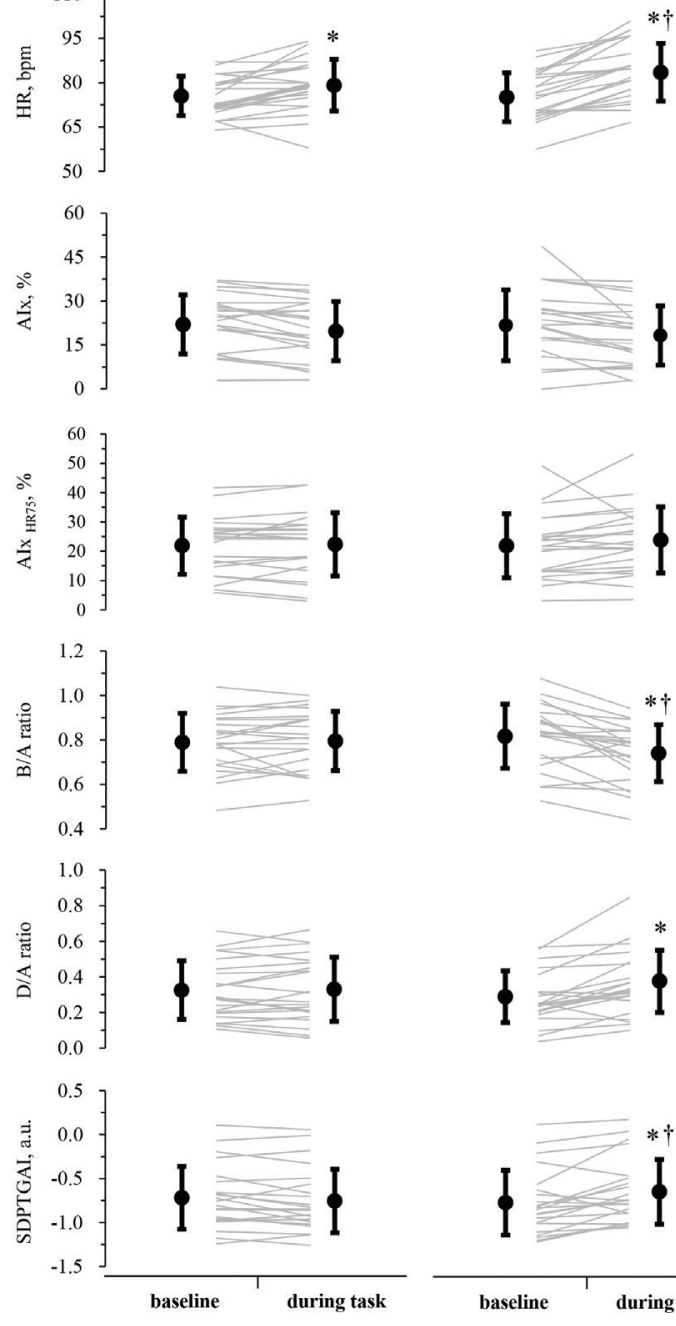

Trial: $\mathrm{P}=0.002$
Time: $\mathrm{P}<0.001$ Time: $\mathrm{P}<0.001$
Interaction: $\mathrm{P}=0.003$
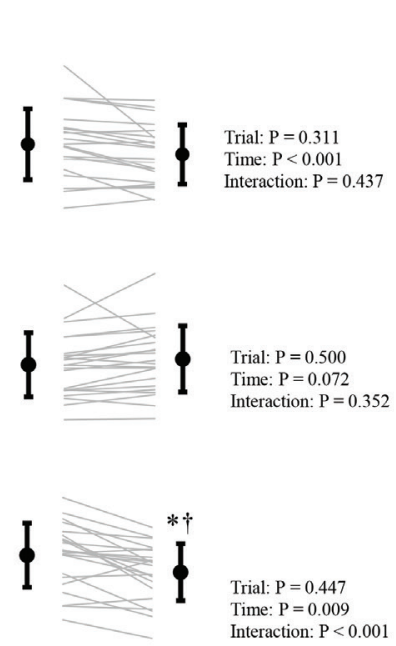

Trial: $P=0.447$ Time: $\mathrm{P}=0.009$
Interaction: $\mathrm{P}<0.001$

Figure 3 Responses of heart rate, Augmentation Index (AIx), and the Second Derivative of the finger Photoplethysmogram (SDPTG) indices to mental arithmetic. $\mathrm{AIx}_{\mathrm{HR} 75}$ indicates AIx adjusted by 75 beat $/ \mathrm{min}$ of heart rate. Gray lines indicate individual data. Closed circles and error bars are mean and SD. ${ }^{*}$ Indicates significant difference from the baseline. ${ }^{\dagger}$ Indicates significant difference with during task in the control condition. a.u. indicates arbitrary unit.

Table 2 Day-to-day reproducibility of pulse rate and the Second Derivative finger Photoplethysmogram (SDPTG) indices

\begin{tabular}{lcccc}
\hline & $\begin{array}{c}\text { Pulse rate, } \\
\text { beat/min }\end{array}$ & $\begin{array}{c}\text { B/A ratio, } \\
\text { ratio }\end{array}$ & $\begin{array}{c}\text { D/A ratio, } \\
\text { ratio }\end{array}$ & $\begin{array}{c}\text { SDPTGAI, } \\
\text { a.u. }\end{array}$ \\
\hline Day 1 $(n=10)$ & $65 \pm 13$ & $-0.81 \pm 0.11$ & $-0.14 \pm 0.09$ & $-0.95 \pm 0.21$ \\
Day 2 $(n=10)$ & $70 \pm 21$ & $-0.81 \pm 0.11$ & $-0.10 \pm 0.10$ & $-0.94 \pm 0.23$ \\
Day 3 $(n=10)$ & $69 \pm 21$ & $-0.86 \pm 0.13$ & $-0.16 \pm 0.15$ & $-0.97 \pm 0.17$ \\
Day 4 $(n=10)$ & $74 \pm 19$ & $-0.82 \pm 0.10$ & $-0.06 \pm 0.10$ & $-1.02 \pm 0.15$ \\
Day 5 $(n=9)$ & $63 \pm 10$ & $-0.83 \pm 0.10$ & $-0.11 \pm 0.10$ & $-1.01 \pm 0.18$ \\
CV, \% $(n=10)$ & $12.5 \pm 5.4$ & $10.6 \pm 5.9$ & $128.7 \pm 139.9$ & $12.0 \pm 9.9$ \\
\hline
\end{tabular}

Data are mean and SD of across all subjects on each day. CV indicates mean and SD of individual the coefficient of variation for 5-day measurement. Abbreviations for SDPTG indices were indicated in Table 1. a.u. indicates arbitrary unit.

group based on the time of measurement before 9:00 am $(n=6$, mean time of day $=7: 35 \mathrm{am})$ or after 11:00 am $(n=4$, mean time of day $=11: 45 \mathrm{am})$, the former group exhibited significantly smaller $\mathrm{CV}$ of the $\mathrm{B} / \mathrm{A}$ ratio $(7.6 \pm 4.5$ vs. $15.0 \pm 5.0 \%, p=0.043)$ and
SDPTGAI $(6.6 \pm 4.4$ vs. $20.1 \pm 10.8 \%, p=0.043)$ than the other groups, whereas no significant group-differences in $\mathrm{CV}$ of pulse rate $(7.6 \pm 4.5$ vs. $14.8 \pm 6.6 \%, p=1.0)$ and $\mathrm{D} / \mathrm{A}$ ratio $(93.9 \pm 66.1$ vs. $181.0 \pm 213.0 \%, p=0.24)$ were observed.

\section{DISCUSSION}

The main findings from this study are twofold: (1) SDPTGAI increased during a short mental arithmetic task (e.g., 30 s) although AIx and $\mathrm{AIx}_{\mathrm{HR} 75}$ remained unchanged; (2) SDPTGAI exhibited excellent reproducibility when it was measured within approximately $10 \mathrm{~min}$, and was positively correlated with AIx. However, the day-to-day variability of SDPTGAI was about $12 \%$. Collectively, these findings suggest that SDPTGAI has the potential for evaluating arterial stiffness and wave reflection, but it should be measured under the well-relaxed resting condition because the finger photoplethysmogram may easily be affected by external stimuli such as mental stress.

Several studies demonstrated clinical and pathophysiological importance of SDPTG indices [4-6]. The B/A ratio, D/A ratio, SDPTGAI can be acquired in a short time and easily by placing a fingertip on the photoplethysmogram sensor. Therefore, this technique has been proposed as a self-monitoring device for healthcare in non-clinical settings such as homes and offices. However, evidence supporting the usability and reliability of SDPTG indices has remained sparse and inconclusive.

Photoplethysmogram optically measures blood volume changes in the microvascular bed of tissue. Since vasoconstriction of microvascular bed may occur instantly with elevation of blood pressure and reduction of skin blood flow during arousing or emotional stress [12], SDPTG may largely be influenced by mental stressor independent of changes in vascular tone and stiffness of the proximal blood vessels. In this study, SDPTG indices changed drastically by a short-term mental stress whereas AIx and its heart rate-adjusted value remained unchanged. Several studies reported that acute mental stress evoked increases in arterial stiffness and wave reflection evaluated by pulse wave velocity and AIx $[10,11]$. Such inconsistency might be due to a duration of mental stress as previous studies conducted 3-5 min of mental arithmetic task. In general, the $\mathrm{B} / \mathrm{A}$ ratio, D/A ratio, and SDPTGAI have been considered as markers of the distensibility of large arteries, the intensity of the reflected wave from the periphery, and vascular aging, respectively [5]. Indeed, at the baseline (resting state), SDPTG indices were strongly correlated with AIx. These results suggest that SDPTG indices partly reflect arterial stiffness and wave reflection under the resting condition.

Augmentation index is affected by changes in heart rate independent of arterial stiffness and wave refection [15]. Of note, the mental arithmetic stimulation increased heart rate but $\mathrm{AIx}$ and $\mathrm{AIx}_{\mathrm{HR} 75}$ did not change significantly. Nevertheless, SDPTG indices significantly altered by the mental arithmetic stimulation. Therefore, alterations in SDPTG indices during acute mental stress is likely to reflect changes in microvascular blood volume and vasoconstriction to a greater extent than arterial stiffness and wave reflection.

In study 1, we obtained ICC of the SDPTG indices and aortic AIx based on the first (e.g., control task) and second (e.g., arithmetic task) baseline values. These measures exhibited excellent reproducibility when they were measured within approximately $10 \mathrm{~min}$. 
On the other hand, the day-to-day variability of $\mathrm{B} / \mathrm{A}$ ratio and SDPTGAI were $10-12 \%$ and comparable to that of pulse rate. The $\mathrm{D} / \mathrm{A}$ ratio showed substantially poor day-to-day variability, shown by $128.7 \pm 139.9 \%$ of CV. This might be attributed to the small height of $\mathrm{D}$ wave which can easily be affected by peripheral vascular tone [5].

Interestingly, SDPTG indices measured after 11:00 am showed significantly larger $\mathrm{CV}$ of the $\mathrm{B} / \mathrm{A}$ ratio and SDPTGAI than the those measured before 9:00 am. These results suggest that SDPTG may have diurnal variation and be influenced by external stimuli such as daily life events and mental stress as shown in study 1 . Therefore, it is recommended that the measurement should be performed under the stress-free environment in a quiet room at comfortable room temperature after at least $10 \mathrm{~min}$ of rest, as recommended by an expert consensus document of aortic stiffness measurement [16]. In addition, to ensure the reliability of the SDPTG indices, the results of repeated measurements (ideally, in the early morning) may need to be referenced.

\section{CONCLUSION}

We determined the usability and reliability of SDPTG indices by comparing between SDPTG indices and AIx at rest and during a short-term mental stress test. We also investigated the day-to-day variability of SDPTG indices. Our findings suggest that SDPTGAI has the potential for evaluating arterial stiffness and wave reflection, but it should be measured under the well-relaxed resting condition because the finger photoplethysmogram may easily be affected by external stimuli such as mental stress. Therefore, to ensure the reliability of the SDPTG indices, the results of repeated measurements (ideally, in the early morning) may need to be referenced.

\section{CONFLICTS OF INTEREST}

The authors declare they have no conflicts of interest.

\section{AUTHORS' CONTRIBUTION}

MF contributed in data collection, data analysis, drafting of the manuscript. TY contributed in data collection. TT contributed in data collection, data analysis, and editing of the manuscript. JS contributed in conceptualization of the study, supervision of the project, data analysis, and editing of the manuscript. All authors contributed in final approval.

\section{ACKNOWLEDGMENT}

The authors deeply thank Ms. Mitsuho Hanamoto for her technical assistance.

\section{REFERENCES}

[1] Vlachopoulos C, Aznaouridis K, Stefanadis C. Prediction of cardiovascular events and all-cause mortality with arterial stiffness: a systematic review and meta-analysis. J Am Coll Cardiol 2010; 55:1318-27.

[2] Vlachopoulos C, Aznaouridis K, Terentes-Printzios D, Ioakeimidis N, Stefanadis C. Prediction of cardiovascular events and all-cause mortality with brachial-ankle elasticity index: a systematic review and meta-analysis. Hypertension 2012;60:556-62.

[3] Takazawa K, Tanaka N, Fujita M, Matsuoka O, Saiki T, Aikawa M, et al. Assessment of vasoactive agents and vascular aging by the second derivative of photoplethysmogram waveform. Hypertension 1998;32:365-70.

[4] Bortolotto LA, Blacher J, Kondo T, Takazawa K, Safar ME. Assessment of vascular aging and atherosclerosis in hypertensive subjects: second derivative of photoplethysmogram versus pulse wave velocity. Am J Hypertens 2000;13:165-71.

[5] Hashimoto J, Watabe D, Kimura A, Takahashi H, Ohkubo T, Totsune K, et al. Determinants of the second derivative of the finger photoplethysmogram and brachial-ankle pulse-wave velocity: the Ohasama study. Am J Hypertens 2005;18:477-85.

[6] Iketani T, Iketani Y, Takazawa K, Yamashina A. The influence of the peripheral reflection wave on left ventricular hypertrophy in patients with essential hypertension. Hypertens Res 2000;23: $451-8$.

[7] Lindqvist M, Melcher A, Hjemdahl P. Hemodynamic and sympathoadrenal responses to mental stress during nitric oxide synthesis inhibition. Am J Physiol Heart Circ Physiol 2004; 287:H2309-H15.

[8] Reims HM, Sevre K, Høieggen A, Fossum E, Eide I, Kjeldsen SE. Blood viscosity: effects of mental stress and relations to autonomic nervous system function and insulin sensitivity. Blood Press 2005;14:159-69.

[9] Lipman RD, Grossman P, Bridges SE, Hamner JW, Taylor JA. Mental stress response, arterial stiffness, and baroreflex sensitivity in healthy aging. J Gerontol A Biol Sci Med Sci 2002;57: B279-B84.

[10] Kume D, Nishiwaki M, Hotta N, Endoh H. Impact of acute mental stress on segmental arterial stiffness. Eur J Appl Physiol 2020;120:2247-57.

[11] Vlachopoulos C, Kosmopoulou F, Alexopoulos N, Ioakeimidis N, Siasos G, Stefanadis C. Acute mental stress has a prolonged unfavorable effect on arterial stiffness and wave reflections. Psychosom Med 2006;68:231-7.

[12] Krogstad AL, Elam M, Karlsson T, Wallin BG. Arteriovenous anastomoses and the thermoregulatory shift between cutaneous vasoconstrictor and vasodilator reflexes. J Auton Nerv Syst 1995;53:215-22.

[13] Sugawara J, Hayashi K, Tanaka H. Distal shift of arterial pressure wave reflection sites with aging. Hypertension 2010;56:920-5.

[14] Kelly R, Hayward C, Avolio A, O’Rourke M. Noninvasive determination of age-related changes in the human arterial pulse. Circulation 1989;80:1652-9.

[15] Wilkinson IB, MacCallum H, Flint L, Cockcroft JR, Newby DE, Webb DJ. The influence of heart rate on augmentation index and central arterial pressure in humans. J Physiol 2000;525 Pt 1: 263-70.

[16] Van Bortel LM, Laurent S, Boutouyrie P, Chowienczyk P, Cruickshank JK, De Backer T, et al. Expert consensus document on the measurement of aortic stiffness in daily practice using carotid-femoral pulse wave velocity. J Hypertens 2012;30:445-8. 